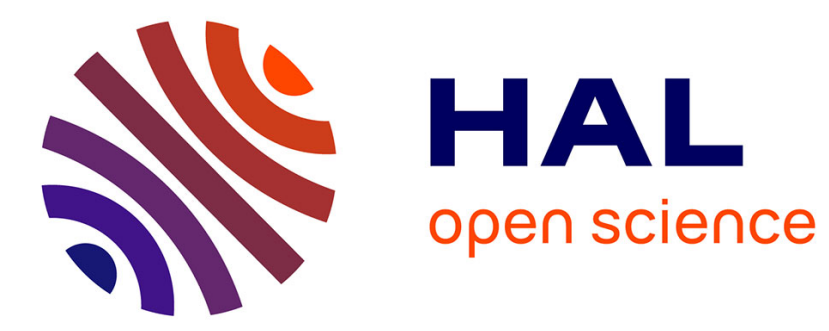

\title{
Improving the Performance of Inventory Systems Subject to Errors by Deploying the RFID Technology
}

\author{
Evren Sahin, Yves Dallery
}

\section{To cite this version:}

Evren Sahin, Yves Dallery. Improving the Performance of Inventory Systems Subject to Errors by Deploying the RFID Technology. Journal of Decision Systems, 2005, 14, pp. N $^{\circ} 4$. hal-00113465

\section{HAL Id: hal-00113465 https://hal.science/hal-00113465}

Submitted on 13 Nov 2006

HAL is a multi-disciplinary open access archive for the deposit and dissemination of scientific research documents, whether they are published or not. The documents may come from teaching and research institutions in France or abroad, or from public or private research centers.
L'archive ouverte pluridisciplinaire HAL, est destinée au dépôt et à la diffusion de documents scientifiques de niveau recherche, publiés ou non, émanant des établissements d'enseignement et de recherche français ou étrangers, des laboratoires publics ou privés. 


\title{
Improving the Performance of Inventory Systems Subject to Errors by Deploying the RFID Technology
}

\author{
Evren SAHIN, Yves DALLERY \\ Laboratoire Génie Industriel \\ Ecole Centrale Paris \\ Grande Voie des Vignes \\ 92295 Châtenay-Malabry, France \\ evren@1gi.ecp.fr \\ dallery@lgi.ecp.fr \\ tel : $\quad 0033141131569$ \\ 0033141131533 \\ fax : $\quad 0033141131272$
}


ABSRACT One of the implicit assumptions considered in the majority of investigations performed in the area of inventory management is that the physical flow that passes through an inventory system as well as the associated information flow are free from defects. In other words, for a given type of product, the quantity received matches exactly the quantity ordered or no errors occur during the data capture process such that the level of the available inventory shown by the information system corresponds exactly to the physical quantity really available. However, various factors can create a difference between the expected and the effective physical and information flows. The performance of such inventory systems whose flows are disturbed by defects can be improved by using advanced product identification and data capture technologies such as the RFID (Radio Frequency Identification) technology. This paper deals with the consideration of errors in inventory management related decisions and analyses whether RFID is cost effective in tackling this issue. We first give an overview of potential errors that may occur within an inventory system. Then, we propose a framework to model the impact of errors and to evaluate the performance improvement enabled by such identification and data capture technologies.

Key words inventory management, product identification and data capture technologies, Radio Frequency IDentification (RFID) technology, errors, Newsvendor problem.

RESUME Une des hypothèses implicites considérées dans le domaine de la gestion de stock est que le flux physique qui traverse un stock ainsi que le flux d'information qui lui est associé ne sont pas sujets à des erreurs. En d'autres termes, pour un type de produit donné, la quantité reçue du fournisseur correspond exactement à la quantité commandée ou des erreurs ne perturbent pas l'enregistrement des données de manière à ce que le niveau de stock indiqué par le système d'information corresponde exactement à la quantité physique disponible. Or, divers facteurs peuvent créer une différence entre le flux physique et le flux d'information prévus et effectifs. Les performances d'un système stock dont les flux sont perturbés par des erreurs peuvent être améliorées par l'utilisation de nouvelles technologies d'identification et de capture de données telles que la RFID (Radio Frequency Identification). Cet article traite de l'impact des erreurs sur la gestion des stocks et analyse si le déploiement de la RFID est justifié. Dans un premier temps, nous fournissons une vue d'ensemble des erreurs qui peuvent impacter un stock. Ensuite, nous proposons un cadre de modélisation pour évaluer l'impact de telles nouvelles technologies d'identification sur la réduction des erreurs et l'amélioration de la performance.

Mots clés gestion de stock, système d'identification de produits et de capture de données, technologie Radio Frequency IDentification (RFID), erreurs, le modèle Newsvendor. 
3 Improving the Performance of Inventory Systems Subject to Errors by Deploying the RFID Technology

\section{Introduction}

Various studies recognize that uncertainties within a supply chain would have substantial impacts on operations (cf. [1], [2], [3]). For instance, [4] states that companies which best cope with uncertainties are most likely to produce internationally competitive bottom-line performances. There are several sources of uncertainties that may lead to inefficiencies within a supply chain. ([5], [6], [7]) provide a review of the notion of uncertainty, which is introduced as being a factor that may generate a variability in quantity, quality or delay, as well as a framework enabling to represent the main factors generating it. They identify several features that may be subject to uncertainties, among which customer demand (even if you know the average demand, there are always variations due to changing customer preferences), physical processes (e.g. fluctuations in process outcomes, in terms of quality, quantity and delays due to variable process yield, machine breakdowns, scrap, unavailable resources, etc.) or data associated with the physical flow that supports decision making. The uncertainty in the last case should be interpreted as a factor that affects one of the four dimensions of data quality which can be described by: (i) data accuracy, i.e. does data reflect exactly the physical system which it is associated with, is it error free and up to date? (ii) data capture delays: what is on average the delay needed to capture data? is this delay subject to variability? (iii) data granularity: at which level data is monitored and managed? (e.g. at product type level or at individual product level) (iv) data availability: can supply chain actors have an easy access to data and is data provided in the right format?

In this paper, we are interested in analyzing the impact of errors that generate an uncertainty on the physical or the information flows, within an inventory system. Typical inventory systems, whether warehouses or stores, are the merge point of thousands of product categories that have different shapes, sizes and colors. Tens of thousands of items may come in and go out the system in a working day [8]. The magnitude of errors (i.e. defects) that perturb the physical flow and the associated information flow can therefore be substantial. For instance, figures found in surveys show that among errors causing perturbations on the physical flow, internal and external theft, administrative errors and fraud made by vendor accounted for $1.8 \%$ of sales in US retail industry in 2001; assuming an annual sales base of \$1.8 trillion, this costs US retailers $\$ 33$ billion [9], European retailers $€ 14.4$ billion, Australasian retailers \$A942 million [10]. Furthermore, Canadian retailers lose approximately $\$ 4.5$ million every single day. For US supermarkets, the National Supermarket Research Group estimates that internal and external theft, receiving errors, damage and retail pricing errors amount to $2.3 \%$ of sales [11].

Similarly, the quality of data that is associated with the physical flow and that supports decision making is one of the biggest problems facing supply chain management ([12], [13]) even when advanced data capture technologies such as the Bar Code system are in use. Several examples illustrate the magnitude of the data inaccuracy problem: [14] reports that the lag from the time a production event occurs until the time it is entered into the system results in the proliferation of bad 
data and the creation of a blank spot in production visibility on the shop floor while [15] states that an average of $30 \%$ of information in retailer systems is incorrect, and studies have shown that as much as $63 \%$ of product descriptions can diverge in supplier and wholesaler systems [16]. In a study conducted for the Grocery Manufacturers of America, A.T. Kearney Inc. estimates that retailers and manufacturers each lose $\$ 2$ million for every $\$ 1$ billion in sales due to bad data. They predict that eliminating bad data could save $\$ 10$ billion per year [17].

In this paper, we consider a supply chain consisting in a manufacturer, a wholesaler and several retailers and we develop a framework that enables to evaluate the impact of the RFID (Radio Frequency IDentification) technology on reducing errors that cause perturbations. RFID is a component of the recent product identification and data capture system (i.e. the Automatic Identification system) developed by the Auto ID Center of MIT that is based on the use of wireless tags carrying EPCs (Electronic Product Codes). RFID readers placed at different points within an inventory system enable to detect products individually and automatically (without human intervention) every time items flow through them and therefore contribute to the elimination of errors. For a detailed comparison between the Bar Code and the RFID technologies shedding light on the advantages of using the RFID system, the reader is referred to [18].

We have noticed that most of research in the field of inventory management make several assumptions such as: (1) entities (individual products and logistical units) remain in perfect condition when they are handled within a supply chain, (2) data gathered from physical transactions is accurate (3) there is a perfect alignment between the movement of products and the associated data records. However, in practice, due to several factors, the effective (physical and information) flow can diverge from the expected flow compromising the validity of these assumptions.

The purpose of this paper is to evaluate the impact of RFID on reducing these factors. The paper consists in several sections; first, section 2 clarifies the main sources of uncertainties by providing an overview of potential errors that may perturb flows, then, section 3 and 4 propose a general framework enabling to represent the different models that can be built in order to evaluate the economic impact of errors by distinguishing between two classes of models, i.e. models with errors and the model without errors. Section 5 pinpoints the improvements that would result from the use of RFID. Section 6 proposes several measures that enable to asses whether the deployment of RFID is economically justified or not. We illustrate this evaluation through several examples. Finally, section 7 concludes the paper.

\section{Overview of Factors Generating Errors within an Inventory System}

Most of earlier investigations on the issue of errors occurring within an inventory system recognizes that these are induced by several factors such as theft, execution or data capture errors. Nevertheless, there has been limited effort on building a more structured approach for a better understanding of the sources of inefficiencies. This 
5 Improving the Performance of Inventory Systems Subject to Errors by Deploying the RFID Technology

section provides an overview of errors that may affect the expected nominal supply chain flows.

\subsection{Errors impacting the physical flow}

As products flow through the supply chain, errors may arise during the different manual hand off steps between manufacturing plants, distribution centers or retail stores and as a result, the effective physical flow would diverge from the expected nominal flow. Within an inventory system, without any anomalies occurring in the expected physical flow, the inventory available would increase each time an expected input arrives (e.g. replenishment from suppliers, customer returns,..) and decrease each time an expected quantity is consumed (e.g. customer demand, known losses of products,..). These movements of inventory can be qualified as known inputs and outputs (cf. figure 1).

Factors that may create defects in this system, qualified as unknown inputs and outputs, may stem from the supply process or they may be internal. Among examples of such factors are suppliers delivering fewer or more products than the quantity ordered; product returns that are not processed properly; products stolen during transportation or storage; undetected perishment or damage; execution problems such as items being placed and forgotten in another location than where they normally should be leading to phantom products or errors done when loading/unloading pallets generating underages or overages in shipments and receipts.

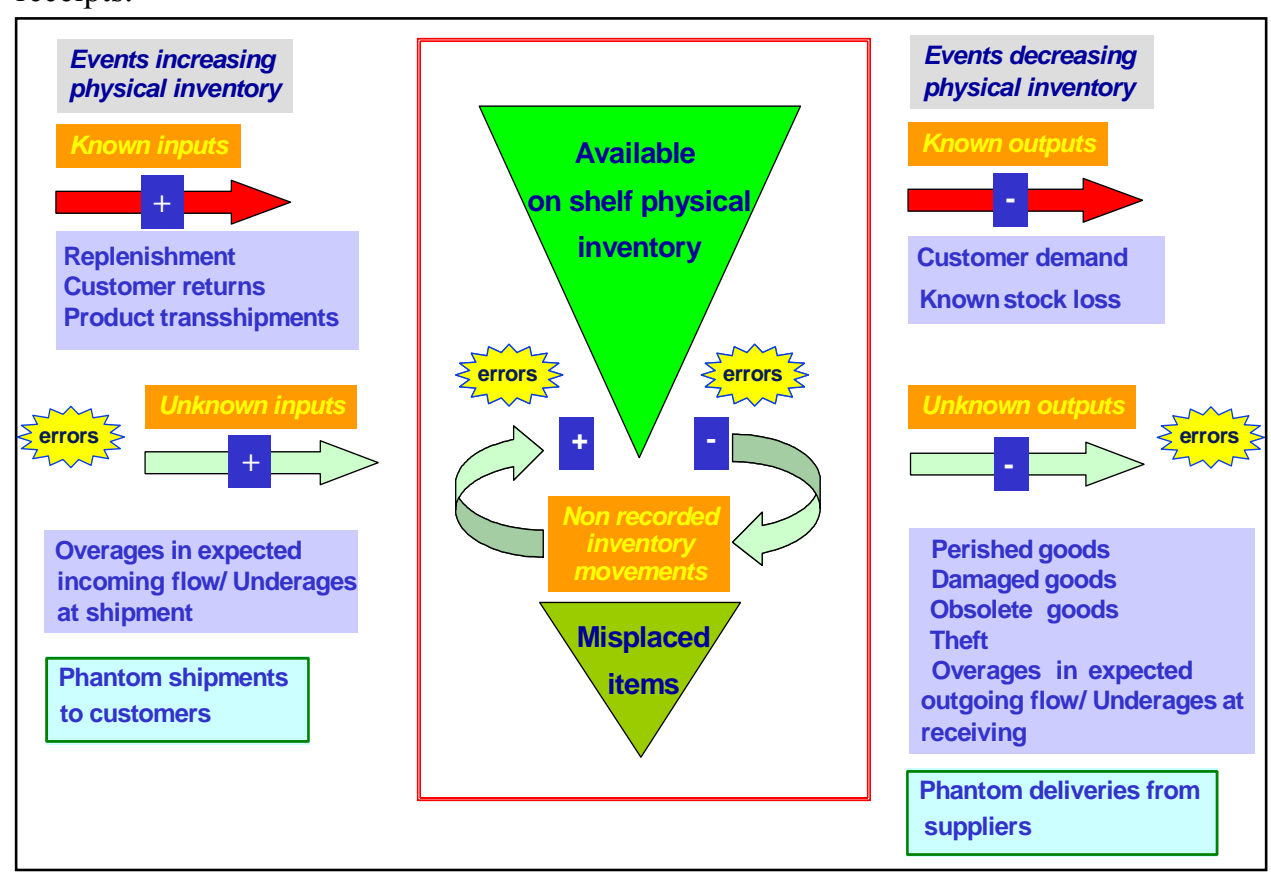

Figure 1. The physical flow within an inventory system 
These unknown factors would cause $\mathbf{I}_{\mathbf{P H}}$, i.e. the effective physical inventory level available within the inventory system, to deviate from I, i.e. the expected nominal on hand inventory level.

Such errors occur from time to time with varying degrees of magnitude. As stated by [10], research in this area is viewed as a data desert, this contributing to the difficulty practitioners experience when getting their issues reviewed at a strategic level. To our knowledge, there are a few industry oriented analyses illustrating these factors and providing estimates of their magnitudes. The only information we found is from [9], [11] and [19] reporting that $0.25 \%$ of items are incorrect in deliveries, $1.5 \%$ of items are stolen in storage and $0.2 \%$ of inventory gets unsaleable. Furthermore, concerning misplaced items, we found in [20] which uses data from the annual physical audits of a company, that, on average, over 6000 SKUs in a store (or $3.4 \%$ of an average store's assortment) are misplaced in storage areas within the store where consumers cannot find or purchase them.

\subsection{Errors impacting the information flow}

In practice, the update of data recorded in the information system (IS) concerning the flow of material coming in and going out of the system can be realised in two ways:

(i) IS data is updated based on measurement: If inventory managers expect that the physical flow to be affected by errors, they will do measurements in order to better streamline it. Data can be gathered either manually or by using a product identification and data collection technology such as the Bar Code system. Even when this technology is used, as stated in [21], the accuracy of data records is still an issue at stake in many industrial practices. The main factors that create divergences between the effective and expected information flows are as follows:

- Errors may arise when collecting data pertaining to known input and output transactions, e.g. (i) scanners may not identify products due to poor symbology specification and coding quality, poor contrast, damaged labels, labels obscured by sealing tape or incorrect check digits [22], (ii) mistakes in the definition of shipping quantities can also generate errors (e.g. shipping one case rather than one unit of a particular SKU), (iii) operators may not distinguish the different variants of an SKU that have similar appearances -e.g. in a store, when a consumer wishes to buy two similar products with identical prices, the cashier often scans only one of the products and treats both products as identical items (iv) operators may forget to scan items' bar code labels or scan them twice (v) for perishable items, since the sell by date information is not carried by bar code labels, the check of best before dates is performed manually: during this activity, a misreading or a faulty identification of the oldest stock results in fresher products being picked ahead of older stock.

- The Bar Code system is a line of sight technology (i.e. a bar code reader has to see the label in the proper orientation for scanning) and as a consequence, items cannot be monitored continuously unless a perpetual scan is being done. Therefore, events 
7 Improving the Performance of Inventory Systems Subject to Errors by Deploying the RFID Technology

that affect the physical inventory may not be detected when they occur: for instance, depending on the scan frequency, employee or customer theft, damage arising during storage or perished items disposed of without inventory corrections being made in IS may continue to deplete the physical inventory while at the same time, their inventory record remains unchanged. For instance, [22] denotes that a scanning exercise carried out by Sainsbury's revealed that some $4 \%$ of traded units which were bar-coded by manufacturers of proprietary brands did not scan at all, while a massive $95 \%$ did not comply with ANA (Article Numbering Association) standards. Only $27 \%$ of the sample of 3,300 traded unit codes tested scanned first time.

For such reasons, the alignment between $\mathbf{I}_{\mathbf{I S}}$, i.e. what the information system shows as available, and $\mathbf{I}$ is compromised.

(ii) IS data is updated automatically based on order information: If managers are not aware of the likelihood for errors occurring in the physical flow or if the scanning operation is too costly, they may decide to increase (or decrease) automatically I without measuring it, based only on information exchanged with other supply chain actors (such as order data, shipment notification,...). It is straightforward that, in this case, there is a risk that $\mathbf{I}_{\mathbf{I S}}$ will not correspond to $\mathbf{I}$.

\subsection{The problem of misalignment between the physical flow and the associated data records}

A combination of errors presented so far makes the reality even more complex: the information on the physical inventory level can be inaccurate since (1) the physical flow may be impacted by errors while no errors arise in the data collection process or, (2) the data capture may be prone to errors while there are no errors that perturb the physical flow or, (3) both flows may be impacted by errors. The sheer volume of record keeping often aggravates the inventory data inaccuracy problem. The magnitude of the problem is illustrated in several studies: for instance, [23] reports that the Naval Supply Depot using the Master Stock Record history of a sample of 714 items from the 20000 line item types stocked there, found that $25 \%$ of the item types had accumulated discrepancies that exceeded 24 units after one year. It also found that the distribution of accumulated errors can be closely approximated by a normal distribution. The study of [20] reveals that a large retailer with annual sales of roughly $\$ 11$ billion from more than 1500 stores worldwide estimates a profit loss of $\$ 32$ million annually due to its inventory record inaccuracy problem. According to a store level analysis, 65\% of nearly 370000 inventory records from 37 stores of a large retailer are inaccurate at the time of the physical inventory audit. [24] conducts a similar analysis: he finds that $36 \%$ of 200000 inventory records sampled from several distribution centers of an organization with a large logistical operation were inaccurate. 


\section{A Framework Enabling to Evaluate the Impact of Errors}

The general framework we propose in order to evaluate the economic impact of errors perturbing the physical and/or the information flows is based on the singleperiod Newsvendor model [25-28]. The supply chain under study includes three stages: the supplier, the wholesaler and retailers, where each actor plays a specific role. The supplier is the actor manufacturing the products, the retailers are the actors selling products to the final consumer and the wholesaler is the intermediate actor that buys products from the supplier and resells them to the retailers. As in the classical Newsvendor model, we are concerned with seasonal (or "fashion") type products, characterized by a short product lifecycle and a short selling season. Typical products that fall into this category are clothes (apparel industry). However, there are many other such products: toys, skis, etc. These products are usually manufactured before the beginning of the season because of long production (or distribution) lead time constraints. We focus on a single product.

Long before the season starts, the wholesaler will have to place a single order $Q$ to the supplier since there is no opportunity for replenishment during the season either because the replenishment lead times are too long or the cost of purchasing products during the season is too high. At this time, the retailers have not yet committed themselves to the wholesaler. Therefore, the choice of $Q$ is based on forecast type information regarding retailers' future demand. When products ordered are received and stored within the wholesaler's inventory, the physical and information flows associated with $Q$ will be as follows:

The physical flow: The physical quantity available within the wholesaler's inventory to satisfy retailers' demand, i.e. $Q_{P H}$, may be different from the quantity ordered (cf. section 2.1.).

The information flow: (1) If data is updated by measuring the effective physical flow (cf. section 2.2.), because of measurement (scan) errors, the available quantity appearing in the information system of the wholesaler, i.e. $Q_{I S}$, would be different from $Q_{P H}$. (2) Alternatively, if the wholesaler assumes that there are no anomalies that occur in the physical flow, he may set $Q_{I S}=Q$ automatically, once the order is placed.

\section{Various Models of Interest}

We can derive two classes of models that belong to the general framework presented above: the model without errors and models with errors. The notations that will be used to describe these models are as follows:

- $\quad C_{0}(Q)$ : The expected total cost in the model without errors

- $Q_{0}{ }^{*}$ : The optimal order quantity in the model without errors

- $C_{0}\left(Q_{0}^{*}\right)$ : The corresponding optimal cost

- $\quad C_{j}(Q)$ : The expected total cost in Model $j$ with errors 
9 Improving the Performance of Inventory Systems Subject to Errors by Deploying the RFID Technology

- $Q_{j}^{*}:$ The optimal order quantity in Model $j$ with errors

- $\quad C_{j}\left(Q_{j}^{*}\right)$ : The corresponding optimal cost

\subsection{The model without errors}

Model 0, which corresponds to the classical Newsvendor model, is the model associated with a perfect situation in which there are no errors occurring, neither regarding the physical flow nor regarding the information flow. The detailed assumptions corresponding to Model 0 are as follows:

Physical quantity: The physical inventory available to satisfy demand when the selling season starts is equal to the quantity ordered $Q$, leading to $Q_{P H}=Q$.

IS inventory data: The IS inventory is obtained by measuring the physical flow using a perfect data capture system, that is, there are no errors involved in this process, or by setting the IS inventory equal to the quantity ordered. In this case, the two approaches are equivalent and lead to $Q_{I S}=Q$.

The behavior of Model 0 is as follows: at the beginning of the season, the wholesaler receives a total demand of $D$ products from the retailers. This demand is addressed in two steps:

- An initial commitment is made by the wholesaler to the retailers based on the IS inventory information. The commitment corresponds to a quantity equal to $\operatorname{Min}(D, Q)$. The corresponding shortage quantity is thus given by $\operatorname{Max}(0, D-$ $\operatorname{Min}(D, Q))$.

- Some time after, products are shipped to the retailers. Because the physical inventory and the IS inventory are identical, the initial commitment is always fulfilled.

If the physical inventory exceeds the shipped quantity, there would be some leftover inventory at the end of the selling period. The corresponding overage quantity is thus given by $\operatorname{Max}(0, Q-\operatorname{Min}(D, Q))$.

The corresponding costs are then given by:

- Overage cost: $h$. $\operatorname{Max}(0, Q-\operatorname{Min}(D, Q))$

- Shortage cost: $\mathrm{u}_{1} . \operatorname{Max}(0, D-\operatorname{Min}(D, Q))$

Where $h$ and $u_{1}$ are respectively the unit overage penalty and the unit shortage (underage) penalty. These parameters can be related to the basic cost parameters $P_{W}, P_{R}$ and $P_{S}$ (which are respectively the (wholesaler) unit product purchasing cost, the unit product selling price, the unit product salvage value) as follows [18]: $h=P_{W}-P_{S} ; u_{1}=P_{R}-P_{W}$. 
The optimization of the system consists in determining the quantity which minimizes the expected total cost, which is the sum of the costs pertaining to these two situations.

\subsection{Models with errors}

Models with errors assume that $Q_{P H}$ is affected by perturbations. These models fall into two categories, depending on the way that $Q_{I S}$ is updated:

- The first type of model, i.e. Model 3, assumes that $Q_{I S}$ is updated based on data collected from the physical flow and that this measurement is subject to errors.

- In the second type of model, i.e. Model 4, we assume that $Q_{I S}$ is set automatically equal to the quantity requested from the supplier when the order is placed.

Note that Model 4 is a special case encountered in facilities which do not scan merchandise during the receiving process. As reported in [29], this situation is encountered by stores that do not verify the incoming products. This allows potential shipment and storage errors to remain invisible. In such cases, shipments from suppliers appear in the receiving store's inventory records once en route. Then, individual items are not scanned upon arrival but rather operators verify the shipment quantity only for items exceeding a particular dollar amount. Had items been stolen from the loading area or en route, packed onto the wrong truck or unloaded at the wrong store, there would be no way for the receiving store to update its inventory records to reflect the actual shipping quantity.

Model 4 will not be analyzed in this study. Our focus is on models where $Q_{I S}$ is updated by measuring (scanning) $Q_{P H}$. First, the generic model with errors, i.e. Model 3, is described while the remaining of the paper examines the behavior of Model 1 which is a special case of Model 3.

The assumptions associated with Model 3 can be described by:

Physical quantity: The physical inventory available to satisfy demand when the selling season starts is different from the quantity ordered $Q$, i.e. $Q_{P H}=Q_{A}$ where $Q_{A}$ is a random variable function of $Q$.

IS inventory data: Due to errors occurring in the data collection process $Q_{I S}=$ $Q_{B}$, where $Q_{B}$ is a random variable function of $Q_{P H}$.

The behavior of models with errors is as follows: at the beginning of the season, the wholesaler receives a total demand of $D$ products from the retailers. This demand is addressed in two steps:

- An initial commitment is made by the wholesaler to the retailers based on the IS inventory information. The commitment corresponds to a quantity equal to $\operatorname{Min}\left(D, Q_{I S}\right)$. The corresponding shortage quantity is thus given by: $\operatorname{Max}\left(0, D-\operatorname{Min}\left(D, Q_{I S}\right)\right)$. 
11 Improving the Performance of Inventory Systems Subject to Errors by Deploying the RFID Technology

- Some time after, products are shipped to the retailers. However, it may happen that it is not possible to fulfill the whole commitment because there are not enough physical products available in the warehouse. This second shortage situation arises if $\operatorname{Min}\left(D, Q_{I S}\right) \geq Q_{P H}$. The corresponding shortage quantity is thus given by: $\operatorname{Max}\left(0, \operatorname{Min}\left(D, Q_{I S}\right)-Q_{P H}\right)$.

If the physical inventory exceeds the shipped quantity, there would be some leftover inventory at the end of the selling period. The corresponding overage quantity is thus given by: $\operatorname{Max}\left(0, Q_{P H}-\operatorname{Min}\left(D, Q_{I S}\right)\right)$.

The corresponding costs are then given by:

- First shortage or shortage type 1 cost: $\mathrm{u}_{1} \cdot \operatorname{Max}\left(0, D-\operatorname{Min}\left(D, Q_{I S}\right)\right)$

- Second shortage or shortage type 2 cost: $\mathrm{u}_{2} \cdot \operatorname{Max}\left(0, \operatorname{Min}\left(D, Q_{I S}\right)-Q_{P H}\right)$

- Overage cost: h. $\operatorname{Max}\left(0, Q_{P H}-\operatorname{Min}\left(D, Q_{I S}\right)\right)$

where $u_{1}, u_{2}$ and $h$ are the unit cost parameters associated with these three penalties respectively. Parameters $h, u_{1}$ and $u_{2}$ can be related to the earlier defined basic cost parameters as follows [18]: $h=P_{W}-P_{S} ; u_{1}=P_{R}-P_{W} ; u_{2}=u_{1}+C_{u_{2}}$ where $C_{U_{2}}$ represents the additional penalty per unit of product that was supposed to be delivered to retailers (based on the wholesaler initial commitment) but failed to be delivered effectively because of missing products discovered just before loading the truck: retailers who do not receive all products they were supposed to are usually upset and often demand a refund or a replacement. Moreover, they may justifiably want the wholesaler to send them the missing products, causing the wholesaler to purchase the products at a higher price during the season.

The optimization of the system consists in determining the quantity which minimizes the expected total cost, which is the sum of the costs pertaining to these three situations.

Remark: While two of the cost components are the ones that already appear in the classical Newsvendor model, the third component is specific to our study: there is a penalty of failing to satisfy a demand after commitment is made for which can be substantially higher than the penalty of initially rejecting this demand.

Notations: Without loss of generality, throughout the paper, we assume that $h=1$, unit cost parameters being given by $\mathrm{k}$ and $\mathrm{m}$ which are respectively defined as $k=\frac{u_{1}}{h}$ and $m=\frac{u_{2}}{u_{1}}$.

\subsection{Variants of Model 3}

Model 3, which is the model the most encountered in practice, takes into account the two types of errors identified so far: errors on the physical flow and on the information flow. However, the simultaneous consideration of defects perturbing 
$Q_{P H}$ and $Q_{I S}$ creates several problems. Firstly, the analysis of such a model is very tedious, if not impossible. Secondly, even if this model could be solved, it would probably be very difficult to interpret the results and get some interesting insights. As a result, we consider two variants derived from Model 3:

- Model 1 which assumes that there are no errors on the physical flow but that data capture errors perturb the information flow, i.e. $Q_{P H}=Q$ and $Q_{I S}=Q_{B}$.

- Model 2 which assumes that the physical flow involves errors but that the data capture process is perfectly reliable, i.e. $Q_{P H}=Q_{A}$ and $Q_{I S}=Q_{P H}$.

Remark: It should be pointed out that either Model 1 or Model 2 should both be interpreted as a special case of Model 3. Indeed, in Model 1, if we were sure that the physical quantity available was always equal to the quantity ordered, there would be no need for gathering data and therefore no errors would be involved in setting the IS inventory quantity. Thus, Model 1 will be used to get insights that should then be thought of as being relevant for the more general Model 3.

The synthesis of the different models discussed in this part is represented in the figure below:

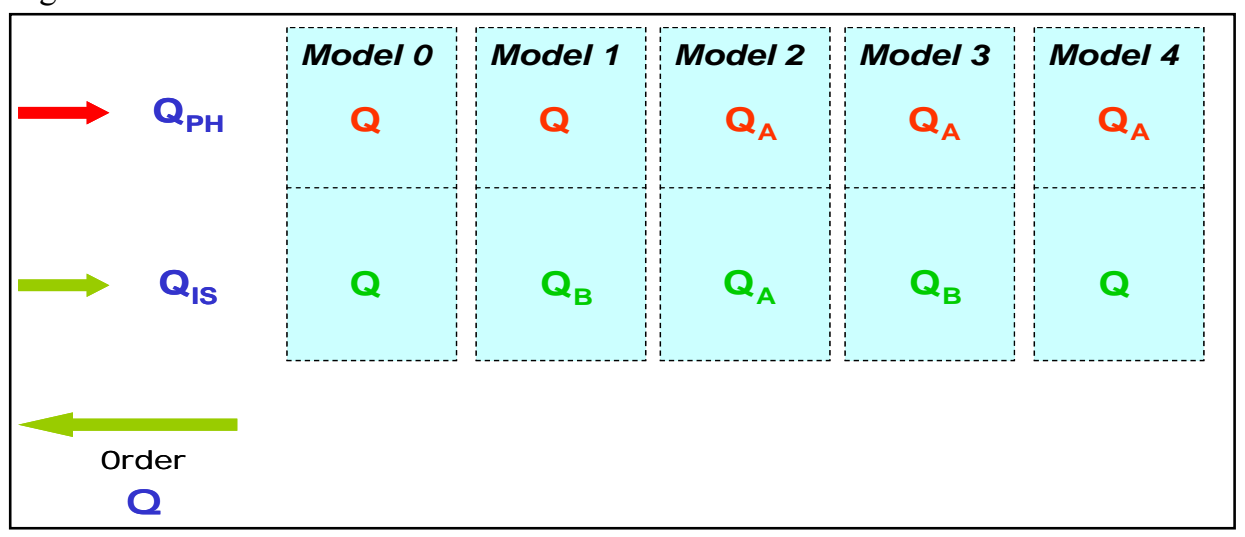

Figure 2. Synthesis of the different models

\subsection{The modelling of errors}

The most general expressions of $Q_{I S}$ and $Q_{P H}$ will respectively be as follows: $Q_{B}=p_{1} \cdot Q_{A}+\varepsilon_{1}$ and $Q_{A}=p_{2} \cdot Q+\varepsilon_{2}$ where $p$ and $\varepsilon$ are random variables with parameters $\left(\mu_{p}, \sigma_{p}\right)$ and $\left(\mu_{\varepsilon}, \sigma_{\varepsilon}\right)$.

The way to determine the parameters pertaining to errors would consist in collecting data and comparing values of $Q_{P H}$ and $Q_{I S}$ observed in various selling seasons to characterize the types and magnitudes of errors. In this paper, we will consider that estimates of these parameters come from prior experience and take them as exogenous. 
13 Improving the Performance of Inventory Systems Subject to Errors by Deploying the RFID Technology

Three special cases can be derived from the general expression presented above (note that we use $Q_{B}$ to illustrate examples below although principles presented here are also valid for $Q_{A}$ )

- Multiplicative errors, i.e. $Q_{B}=p \cdot Q_{A}$ where $\mathrm{p}$ is a non negative random variable with parameters $\left(\mu_{p}, \sigma_{p}\right)$. This leads to $E\left[Q_{B}\right]=\mu_{p} \cdot Q_{A}$ and $\sigma\left[Q_{B}\right]=\sigma_{p} \cdot Q_{A}$, dependent on $Q_{A}$.

- Additive errors, i.e. $Q_{B}=Q_{A}+\varepsilon$ where $\varepsilon$ is a random variable with parameters $\left(\mu_{\varepsilon}, \sigma_{\varepsilon}\right)$. This leads to $E\left[Q_{B}\right]=\mu_{\varepsilon}+Q_{A}$ and $\sigma\left[Q_{B}\right]=\sigma_{\varepsilon}$, independent of $Q_{A}$.

- Mixte errors, in between these two models, the third alternative for modelling errors is to set $Q_{B}=p \cdot Q_{A}+\varepsilon$ with $\sigma_{p}=0$ and $\mu_{\varepsilon}=0$ which leads to $E\left[Q_{B}\right]=\mu_{p} \cdot Q_{A}$ and $\sigma\left[Q_{B}\right]=\sigma_{\varepsilon}$.

\section{Remarks}

1. A hypothesis that is less realistic consists in assuming that errors are deterministic, i.e. $Q_{B}=\mu_{p} \cdot Q_{A}$ or $Q_{B}=\mu_{\varepsilon}+Q_{A}$ where $\mu_{p}$ and $\mu_{\varepsilon}$ are supposed to be known. This assumption enables to simplify the formulation and the optimisation of the expected cost function.

2. Assuming that errors are such that the quantity observed in IS has the same probability to be more or less than the physically available quantity is representative of what is usually found in practice.

It may also be interesting to consider extreme situations where errors are one sided i.e. case 1: errors are such that $Q_{P H}$ is totally underestimated, i.e. $Q_{B} \leq Q_{A}$ and case 2: errors are such that $Q_{P H}$ is totally overestimated, i.e. $Q_{A} \leq Q_{B}$. This would enable to take into account the effect of positive and negative discrepancies separately. This renders the formulation of the expected cost less complex than the non biased case (i.e. $\mu_{\varepsilon}=0$ for the additive case and $\mu_{p}=1$ for the multiplicative case).

The behaviours of cases 1 and 2 are not symmetrical, since in case 1, a shortage type 2 penalty is never incurred. Furthermore, one can notice that the expected cost is composed of two parts: the first part expresses the cost incurred by the wholesaler when a random quantity $\mathrm{Q}_{\mathrm{B}}$ is received for each order $\mathrm{Q}$ placed to the supplier. The second part expresses the additional overage penalty stemming from having a quantity $\mathrm{Q}_{\mathrm{A}}$ in stock that is not used to satisfy demand since not recorded in IS. We deduce therefore that this model is an adjusted random yield problem. The reader is referred to [30] for further details.

One can also remark that if $\mathrm{u}_{2}=\mathrm{u}_{1}$ the model associated with the case $Q_{A} \leq Q_{B}$ is equivalent to a classical Newsvendor problem. 


\section{Impact of the deployment of the RFID technology}

RFID is demonstrating measurable results that have the potential to transform how business is conducted in supply chains. The benefits of deploying RFID at item level within an inventory system can be divided in two categories:

- Impact on the information flow: this advanced technology allows an accurate capture of data associated with the physical flow. Depending on the initial situation considered, this impact can be quantified by comparing:

- the optimal cost associated with Model 4 to the optimal cost associated with Model 2

- the optimal cost associated with Model 3 to the optimal cost associated with Model 2

- the optimal cost associated with Model 1 to the optimal cost associated with Model 0

These comparisons enable to quantify the improvements resulting from a more accurate IS inventory data given the existing physical flow of products.

- Impact on the physical flow: coupled with other processes supporting it, the RFID technology contributes to the elimination of errors that cause perturbations in the physical flow. The elimination of theft (by discouraging thieves or determining the status of the product -purchased or not purchased- at customer service desk in order to eliminate fraudulent store returns) and a faster detection of perished items (by facilitating better FIFO and promotions management or by improving data integrity and visibility in order to permit staff to select the oldest product first to reduce perishable product waste) can be cited among such contributions.

The impact of RFID on the physical flow can be quantified by comparing the optimal cost associated with Model 2 to the optimal cost associated with Model 0.

In situations where errors impacting the physical flow stem from the supplier, the benefit of the use of RFID at the supplier level can be evaluated by comparing the optimal cost associated with Model 3 to the optimal cost associated with Model 1.

The scheme below represents these two impacts simultaneously: 
15 Improving the Performance of Inventory Systems Subject to Errors by Deploying the RFID Technology

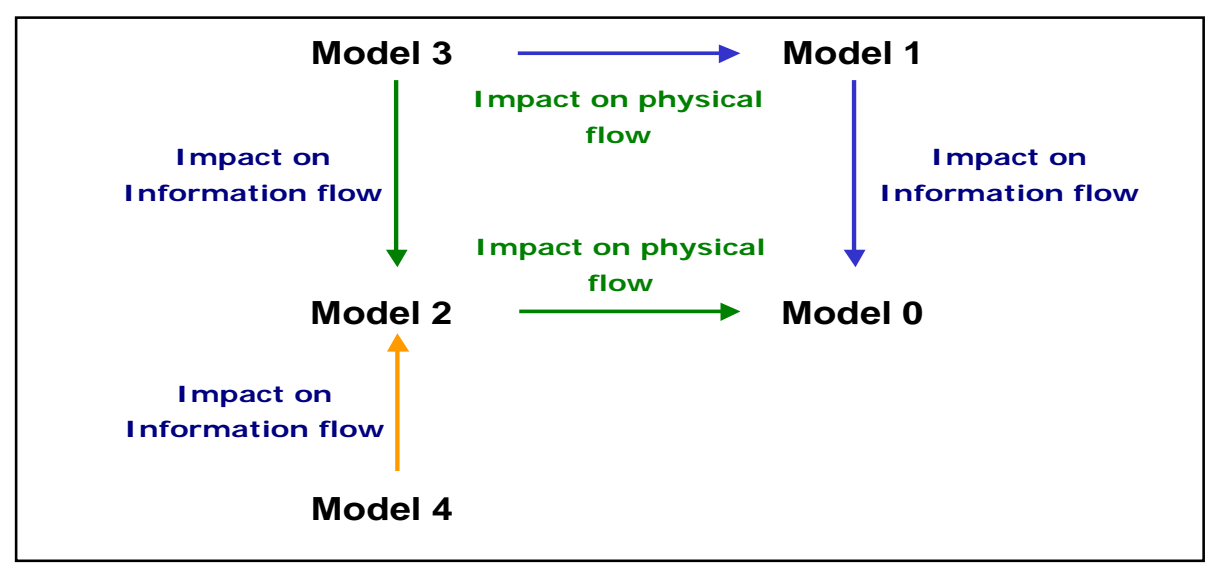

Figure 3. Impacts of the RFID technology

\section{Evaluation of the benefit of eliminating errors}

Whether defects perturb the physical flow or the data collection process is prone to errors, the result is the occurrence of additional inventory related costs. If we consider an initial situation where errors exist and if the decision system does not consider the likelihood for these errors creating divergences from the expected nominal flows, it would act as if there were no defects and decide to order $Q_{0}^{*}$. The cost incurred will be $C_{j}\left(Q_{0}^{*}\right)$.

The potential benefit that would stem from improving this system, i.e. moving from an imperfect situation (Model j) to a perfect error free situation captured by Model 0 can be measured by using the following indicator: $R_{3}=\frac{C_{j}\left(Q_{0}^{*}\right)-C_{0}\left(Q_{0}^{*}\right)}{C_{j}\left(Q_{0}^{*}\right)}$

We think that it is interesting to track the evolution of this ratio since we believe that, most companies ignore the existence of errors and operate actually at $C_{j}\left(Q_{0}^{*}\right) . C_{0}\left(Q_{0}^{*}\right)$ being the baseline to assess savings resulting from eliminating errors, $\mathrm{R}_{3}$ takes into account the additional cost generated by defects in information and/or physical flow within a facility.

While this ratio represents the total benefit that would stem from improving an inventory system prone to errors, a more detailed analysis that splits this benefit in two parts is also of interest. As represented in the figure below, there are two ways to improve such an inventory system: 


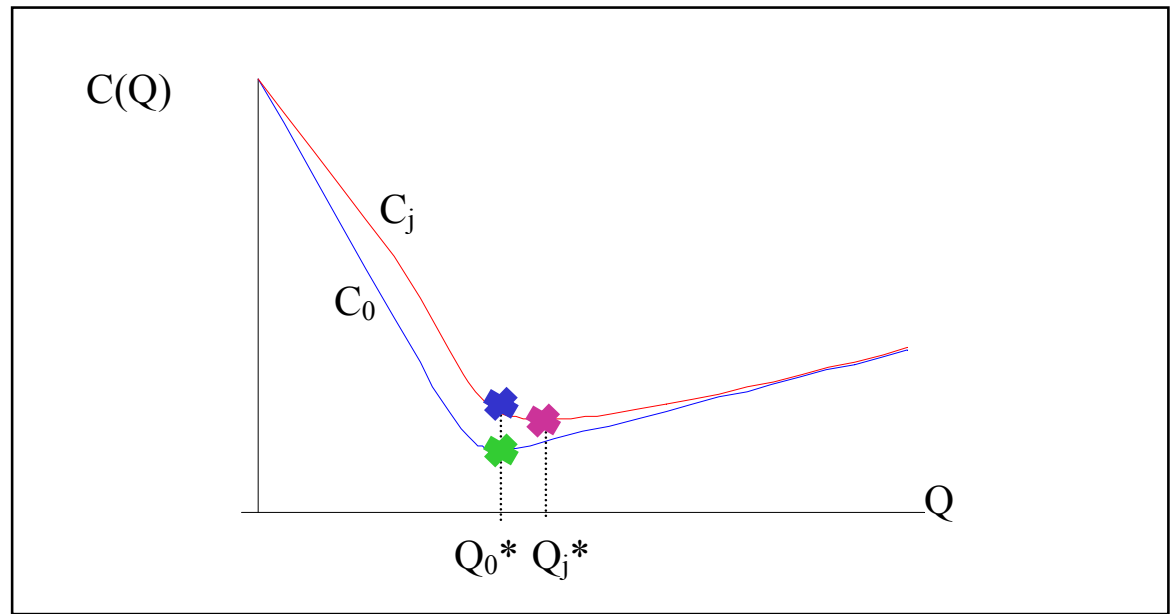

Figure 5. Costs associated with the model with errors and a model without errors

\subsection{Improving performance by taking into account errors when ordering}

If managers are aware of errors and if it is possible to estimate the parameters pertaining to errors, inventory related costs could be reduced by optimizing the system, i.e. by ordering $Q_{j}^{*}$ instead of $Q_{0}^{*}$. The cost incurred will be $C_{j}\left(Q_{j}^{*}\right)$.

Therefore, the penalty due to an inappropriate order quantity resulting from a poor knowledge of the inventory system can be estimated by $C_{j}\left(Q_{0}^{*}\right)-C_{j}\left(Q_{j}^{*}\right)$. In our analyses, we will use the ratio $R_{1}=\frac{C_{j}\left(Q_{0}^{*}\right)-C_{j}\left(Q_{j}^{*}\right)}{C_{j}\left(Q_{0}^{*}\right)-C_{0}\left(Q_{0}^{*}\right)}$ to evaluate the part of the total benefit which is achieved thanks to the optimization of the system by considering the existence of errors when ordering.

\subsection{Improving performance by deploying RFID}

Once the optimization in presence of errors is realized, the cost can be further reduced by deploying appropriate actions eliminating errors such as the implementation of RFID (cf. section 5). Examples of some other actions using a range of people, process and technologies are as follows: re-engineer the physical organization of the warehouse, use a new product identification technology that reduces scanning errors, use a technology that enables to reduce theft in the warehouse, use a technology to effectively track products' sell by dates, double check receiving and shipment processes, improve the actual processes (by defining 
17 Improving the Performance of Inventory Systems Subject to Errors by Deploying the RFID Technology

new working procedures for operators, labour resource priorities and more appropriate indicators to evaluate their performance), do benchmarking analysis and develop personnel awareness building actions that focus on the operational weaknesses, etc. The performance of each action plan, i.e. its contribution to eliminate a certain type of error, as well as the cost of implementing it would vary. For instance, different means could be used to reduce (or even eliminate) the issue of theft: the use of EAS (Electronic Article Surveillance) tags coupled with processes supporting them or better defined operators' working procedures coupled with more frequent verification processes or the use of RFID technology coupled with processes supporting it, etc.

Since the impact of actions eliminating errors can be estimated by $C_{j}\left(Q_{j}^{*}\right)-C_{0}\left(Q_{0}^{*}\right)$, we define a ratio complementary to $R_{1}$, i.e. $R_{2}=\frac{C_{j}\left(Q_{j}^{*}\right)-C_{0}\left(Q_{0}^{*}\right)}{C_{j}\left(Q_{0}^{*}\right)-C_{0}\left(Q_{0}^{*}\right)}$ to evaluate the percentage of the total benefit which is achieved if an action such as the deployment of RFID is performed to eliminate errors.

Note that although we assume in our analysis that RFID is $100 \%$ reliable which enables to completely eliminate errors (an assumption which is not always verified by actual practices), our analysis can be extended to the case where errors are only reduced when RFID is used. Hence, the benefit of RFID will be evaluated by comparing two cases where in the second case, values of error parameters $\left(\mu_{p}, \sigma_{p}\right)$ are smaller.

\subsection{Numerical example}

Aiming at illustrating concepts introduced previously, in this section, we examine one of the models with errors, i.e. Model 1, and compare it with the base case Model 0 in order to evaluate the savings that would result from eliminating errors and to get further insights about conditions under which this savings is the highest. This analysis is a piece of a greater effort (developed in [18]) that examines the performance of inventory systems subject to errors.

All analyses in this section assume that (i) errors are multiplicative, (ii) demand and errors are normally distributed with parameters $\left(\mu_{D}, \sigma_{D}\right)$ and $\left(\mu_{p}, \sigma_{p}\right)$ respectively. In the first example below, we consider the variation of $R_{3}$ with respect to $\sigma_{p}$, the second example focuses on the evolution of $R_{3}$ with respect to $\mu_{p}$ and finally, the third example analyses the split of the expected benefit. The entire study and insights are based on numerical studies, the main reason for this lying in the mathematical complexity associated with the expected 
cost function of Model 1 (cf. appendix 1) and the complexity to interpret results obtained.

Example 1: The figure below assumes that $k=2 ; \mu_{p}=1 ; \mu_{D}=10 ; \sigma_{D}=3$ and evaluates the sensitivity of $R_{3}$ to varying values of $m$.

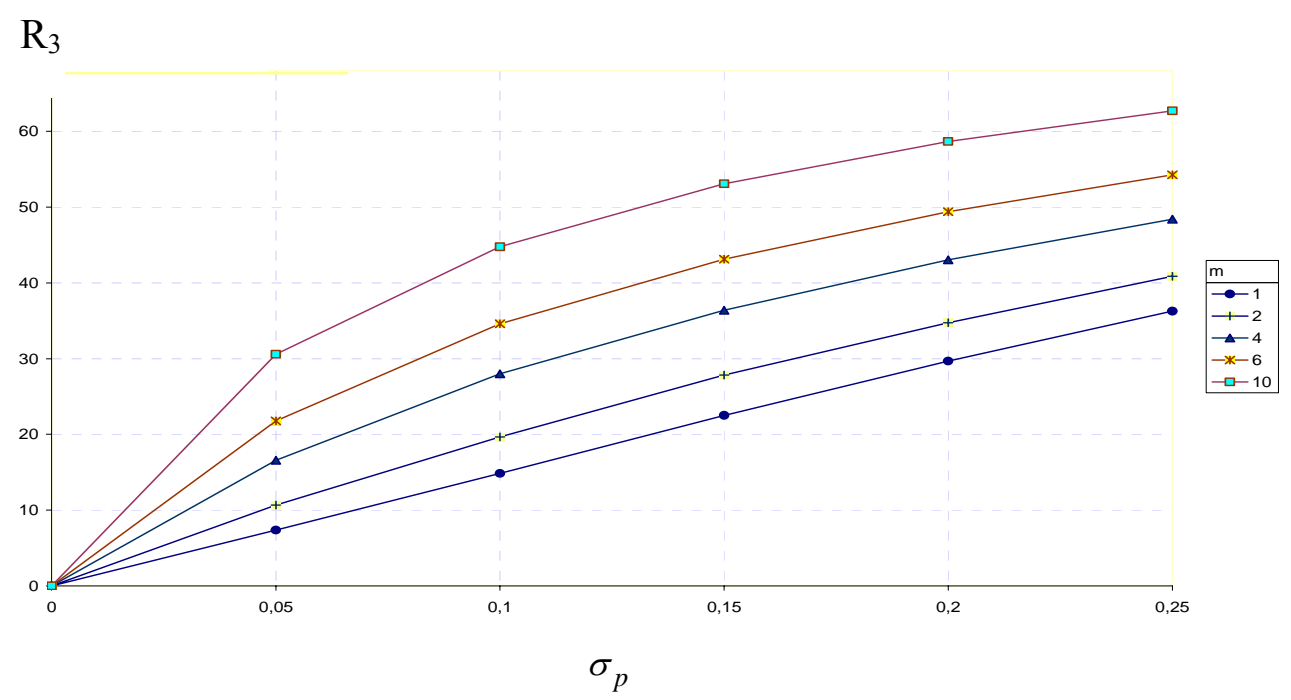

By ignoring errors, one would order $Q_{0}^{*}=11$. If we assume that on average $\mathrm{Q}_{\mathrm{IS}}=\mathrm{Q}_{\mathrm{PH}}$ with a dispersion of $\sigma_{p}=0.05$, demand will be satisfied based on an observed $Q_{I S}$ value that is within the interval $[9,13]$. This will result in an additional relative cost of $7 \%, 11 \%$ and $17 \%$ if $\mathrm{m}$ is respectively equal to 1,2 and 4 .

Further observations pertaining to the evolution of $R_{3}$ are as follows: (i) for a given value of $\mathrm{m}, R_{3}$ increases as $\sigma_{p}$ increases, (ii) for a given value of $\sigma_{p}, R_{3}$ increases as $\mathrm{m}$ increases, since the penalty of observing a shortage type 2 situation is higher.

Managerial Insights: (1) When nothing is done to correct inaccuracies, even for small error rates (in terms of $\sigma_{p}$ ), the penalty of ordering an inappropriate quantity can be substantial. For given values of $\mathrm{k}$ and $\mathrm{m}$, the higher is the variability of errors, the larger will be the penalty of ignoring errors. What is also of interest is how $\mathrm{R}_{3}$ evolves in $\sigma_{p}$ : while for small values of $\mathrm{m}, \mathrm{R}_{3}$ is almost linear in $\sigma_{p}$, for higher values of $\mathrm{m}$, the ratio is more sensitive to $\sigma_{p}$ : e.g. for $\sigma_{p}=0.05, \mathrm{R}_{3}=7 \%$ if $\mathrm{m}=1$ and is up to $30 \%$ if $\mathrm{m}=10$. (2) Furthermore, for a given value of $\sigma_{p}, R_{3}$ increases as $m$ increases: in a context where resources are limited, efforts should be 
19 Improving the Performance of Inventory Systems Subject to Errors by Deploying the RFID Technology

deployed in priority for improving the inventory data accuracy of product categories which are the most affected by the shortage type 2 penalty while planning the roll out of RFID technology.

Example 2: We suppose that $k=2 ; \sigma_{p}=0.05 ; \mu_{D}=10 ; \sigma_{D}=3$ and evaluate the sensitivity of $\mathrm{R}_{3}$ to different values of $\mathrm{m}$.

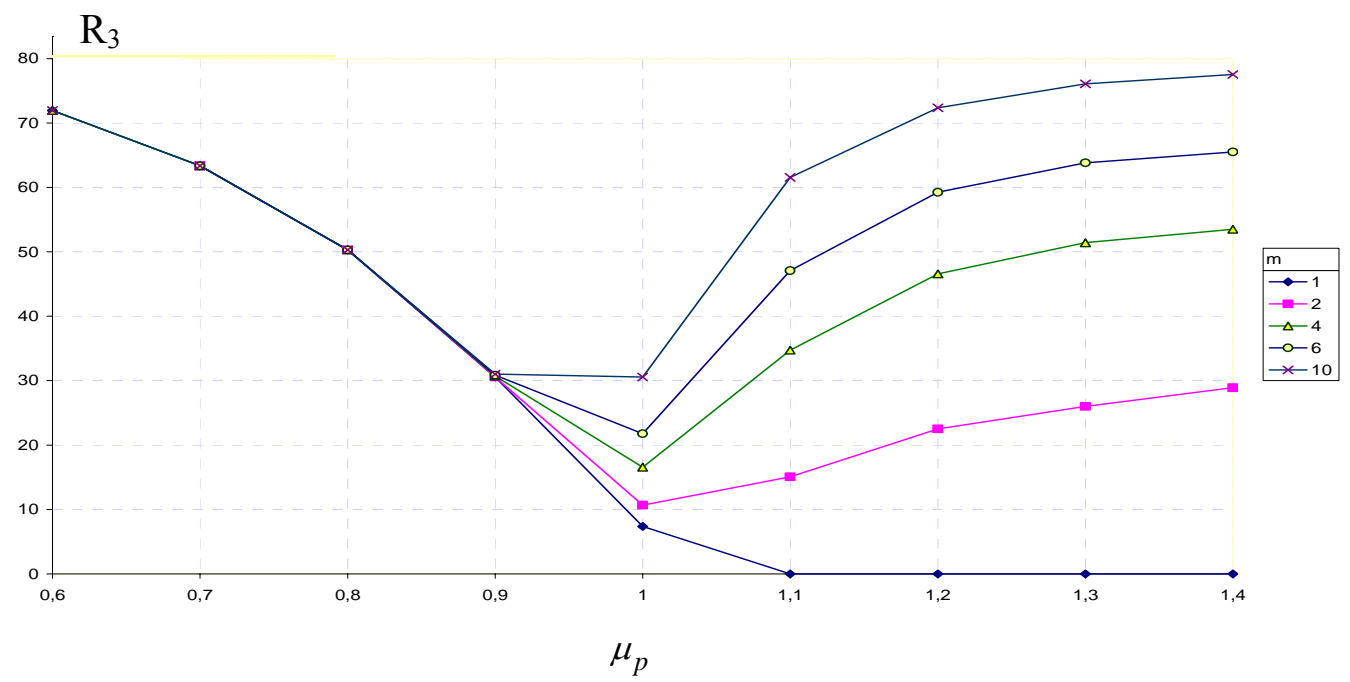

For $\mu_{p}<1, R_{3}$ decreases as $\mu_{p}$ increases: as the reliability of the data capture process is improved, the penalty of ordering $Q=Q_{0}^{*}$ is reduced. If $\mu_{p}$ takes a value higher than a critical value (which depends on parameters $\sigma_{p}, \sigma_{D}, \mathrm{k}, \mathrm{m}$ ), since the shortage type 2 penalty is larger, $R_{3}$ increases as $\mu_{p}$ increases. The value of $R_{3}$ that is associated with this critical value of $\mu_{p}$ where the impact of errors is minimal can be used as a reference point: in situations where parameters pertaining to errors cannot be measured precisely, comparing this value to the cost of implementing RFID would give a first approximation on whether deploying this technology yields a positive net benefit.

Remark: An exception is observed for $m \approx 1$ for which observing $\mathrm{Q}_{\mathrm{IS}}>\mathrm{Q}_{\mathrm{PH}}$ does not generate an additional penalty and as a result, $R_{3}$ is totally decreasing in $\mu_{p}$.

Managerial Insight: Our model explicitly captures the penalty of not satisfying an earlier commitment: the wholesaler incurs a shortage type 2 cost for each unit of product initially promised to retailers but not delivered. In cases where inventory errors underestimate the physical quantity, reducing errors by a given percentage yields almost the same savings for products having different values of $\mathrm{m}$. At the other extreme, if the physical quantity is overestimated, the magnitude of the savings 
depends on the value of m: ordering $Q=Q_{0}^{*}$ has severe consequences especially for products for which $\mathrm{m}$ is high.

Example 3: In the figure below, assuming that $P_{W}=5 \$ ; P_{R}=15 \$ ; P_{S}=0 \$ ; \mu_{D}=10 ; \sigma_{D}=3$, we represent the evolution of $C_{0}\left(Q_{0}^{*}\right), C_{1}\left(Q_{1}^{*}\right)$ and $C_{1}\left(Q_{0}^{*}\right)$ with respect to $\sigma_{p}$ for different values of $\mathrm{m}$.

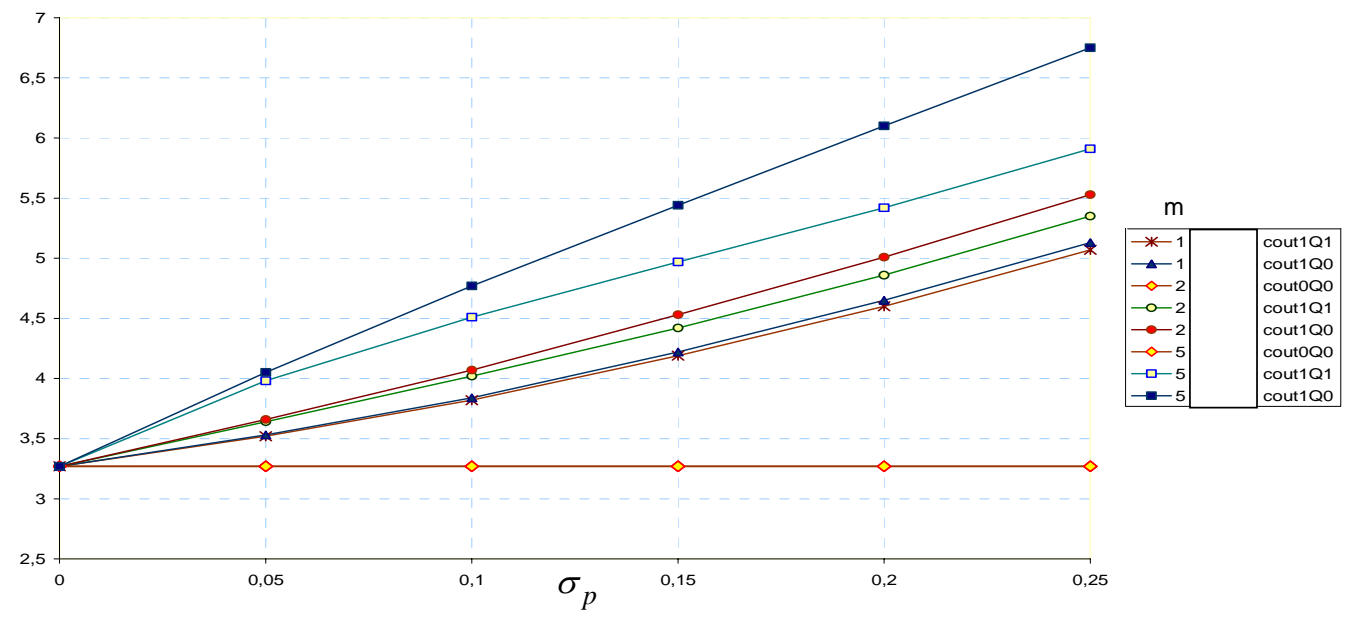

For these parameters, without errors, one would incur $C_{0}\left(Q_{0}^{*}\right)=3.27$. If we assume that, on average $Q_{I S}=Q_{P H}$ i.e. $\mu_{p}=0$ with a dispersion of $\sigma_{p}=0.1$, ordering as if there were no errors generates an additional relative cost (in comparison with $C_{0}\left(Q_{0}^{*}\right)$ ) of $17.43 \%, 24.46 \%$ and $45.87 \%$ if $C_{u_{2}}$ is respectively equal to 0 (i.e. $\mathrm{m}=1$ ), 10 (i.e. $\mathrm{m}=2$ ) and 40 (i.e. $\mathrm{m}=5$ ) . Taking into account the probability for errors when ordering would enable to lessen this additional relative cost to $16.81 \%$, $22.93 \%, 37.92 \%$ for the same values of $C_{u_{2}}$.

Managerial Insight: Even if it can be difficult to be realised in practice, getting information on characteristics (mean and variability) of errors occurring in the data capture process enables to reduce the cost incurred by adjusting the quantity ordered so that it takes into account the likelihood for errors. The comparison of the relative values earned from a better knowledge of errors and the implementation of RFID leads to the following results: (1) for given values of $\mathrm{k}$ and $\mathrm{m}$, as $\sigma_{p}$ increases, the first part of the savings, $C_{1}\left(Q_{0}^{*}\right)-C_{1}\left(Q_{1}^{*}\right)$, increases relatively more than the second part of the savings, $C_{1}\left(Q_{1}^{*}\right)-C_{0}\left(Q_{0}^{*}\right)$, (2) for given values of $\mathrm{k}$ and $\sigma_{p}$, as 
21 Improving the Performance of Inventory Systems Subject to Errors by Deploying the RFID Technology

$\mathrm{m}$ increases, the augmentation of $C_{1}\left(Q_{0}^{*}\right)-C_{1}\left(Q_{1}^{*}\right)$ is relatively larger (in comparison with $\left.C_{1}\left(Q_{1}^{*}\right)-C_{0}\left(Q_{0}^{*}\right)\right)$. In situations where $\mathrm{m}$ and $\sigma_{p}$ are large, the first part of the savings may even be larger than the second part.

\section{Conclusion}

Our starting point was that although supply chain scholars very often assume the availability of error free flows to implement a particular inventory policy, this should not be taken for granted. The uncertainty stems mainly from factors that lead to perturbations that impact the physical flow of products on the one hand, and errors polluting the data capture, on the other hand, even when advanced identification technologies like the Bar Code system are in use. In this work, we separated these two factors and presented in a structured way the root causes, magnitudes and characteristics of errors.

Our work proposes a set of single period inventory models to asses the benefit of using RFID to eliminate errors for seasonal products. Further investigation such as the extension of the work to consider the case of products that can be reused from one period to another within a multi period inventory model setting is now under consideration. The transition to a multi period model necessitates the consideration of several aspects:

In a multi period setting, one can discover that $\mathrm{I}_{\mathrm{IS}}$ and $\mathrm{I}_{\mathrm{PH}}$ are not equal under some circumstances. For instance, a picking operator may detect that $\mathrm{I}_{\mathrm{PH}}<\operatorname{Min}\left(\mathrm{I}_{\mathrm{IS}}\right.$, $D$ ) while preparing an order or a store operator may remark that $\mathrm{I}_{\mathrm{PH}}<\mathrm{I}_{\mathrm{IS}}$ when stock out situations occur. At the other extreme, because of lacking space, an operator replenishing shelves may notice that $\mathrm{I}_{\mathrm{PH}}>\mathrm{I}_{\mathrm{IS}}$. The likelihood of observing these unexpected situations as well as what should be done when they occur need to be considered when designing a particular inventory policy.

Another issue concerns the divergence between $\mathrm{I}_{\mathrm{PH}}$ and $\mathrm{I}_{\mathrm{IS}}$. This issue is discussed by [8] who considers an (r,Q) policy and assumes that the exact value of $\mathrm{I}_{\mathrm{PH}}$ is not known due to a random loss of stock leading to $\mathrm{I}_{\mathrm{PH}}<\mathrm{I}_{\mathrm{IS}}$. In this system, a continual rise between $\mathrm{I}_{\mathrm{PH}}$ and $\mathrm{I}_{\mathrm{IS}}$ is observed and upon a certain point, the system reaches a freezing point where no order is placed since $\mathrm{I}_{\mathrm{IS}}>\mathrm{r}$ although $\mathrm{I}_{\mathrm{PH}}=0$.

Although we did not consider explicitly the cost of manually scanning products to collect inventory data, the cost associated with this operation can be substantial in facilities storing thousands of products and needs to be integrated into the trade off analysis between the additional overage and shortage costs stemming from errors and the cost of counting inventory. 


\section{References}

1. Wilding, R.; The supply chain complexity triangle: uncertainty generation in the supply chain; International Journal of Physical Distribution \& Logistics Management; 1998; Vol. 28(8): p. 599-616.

2. Davis, T.; Effective supply chain management; Sloan Management Review; 1993; Vol. Summer: p. 35-46.

3. Persson, G.; Logistics process redesign: some useful insights; International Journal of Logistics Management; 1995; Vol. 6(1): p. 13-25.

4. Mason-Jones, R. and Towill, D.R.; Shrinking the supply chain uncertainty circle; Control; 1998(September): p. 17-22.

5. Van der Vorst, J. and Beulens, A.; Identifying sources of uncertainty to generate supply chain redesign strategies; International Journal of Physical Distribution \& Logistics Management; 2002; Vol. 32(6).

6. Van der Vorst, J., et al.; Supply Chain Management in Food Chains: Improving Performance by Reducing Uncertainty; International Transactions in Operational Research; 1998; Vol. 5(6).

7. Landeghem, G. and Vanmaele, H.; Robust planning: a new paradigm for demand chain planning; Journal of Operations Management; 2002; Vol. 319.

8. Kang, Y.; Information inaccuracy in information systems; 2003; Ph.D. Dissertation, MIT.

9. Hollinger R.C. and Davis J .L.; National Retail Security Survey Report; Department of Sociology and the Centre for Studies in Criminology and Law, University of Florida; 2001.

10. Alexander, K., et al.; Applying Auto-ID to Reduce Losses Associated with Shrink; Auto ID Center.

11. Supermarket Shrink Survey; National Supermarket Research group; 2001; www. Trax-usa.com/research/home2.html.

12. Kärkkäinen, M. and Holmström, J.; Wireless product identification: enabler for handling efficiency, customisation and information sharing; Supply Chain Management: An International Journal; 2002; Vol. 7(4).

13. Tuttle, A.; Who do you trust; Industrial Distribution; 2002; Vol. 91(3).

14. Hurwitz, D.; Data capture for MRP-II systems; Society of Manufacturing Engineers; 1985.

15. UCCnet; The Value of Clean Data; 2003; http://knowledgebase.uccnet.org/press-Room/publications.

16. Vermeer, B.H.P.J.; Data Quality and Data Alignment in EBusiness; 2001; Eindhoven; Eindhoven University Press Facilities.

17. Mullaney, T.J. and Coy, P.; This Gift Just Keeps On Giving; in BusinessWeek; 2003.

18. Sahin, E.; A qualitative and quantitative analysis of the impact of the Auto ID technology on the performance of supply chains; 2004; Ph.D. Thesis, Ecole Centrale Paris; Paris. 
23 Improving the Performance of Inventory Systems Subject to Errors by Deploying the RFID Technology

19. Lightburn A.; 2002 Unsealables benchmark report; Joint Industry Insaleables Committee, Foods Marketing Institute of Grocery Manufacturers of America.

20. Raman, A., DeHoratius, N., and Ton, Z.; Execution:the missing link in retail operations; California Management Review; 2001; Vol. 43.

21. Alexander, K., et al.; Focus on Retail: Applying Auto-ID to Improve Product Availability at the Retail Shelf; Auto ID Center.

22. Marcel, B.; Verify at source and train staff; Logistics Information Management; 1995; Vol. 8(3).

23. Morey, R.C.; Estimating Service Level Impacts from changes in cycle count, buffer stock or corrective action; Journal of Operations Management; 1985; Vol. 5(4).

24. Millet, I.; A Novena to Saint Anthony, or How to Find Inventory by Not Looking; Interfaces; 1994; Vol. 24(2).

25. Nahmias, S.; Production and Operations Analysis, Fourth Edition; Mc Graw-Hill Irwin; 2001.

26. Silver, E.A., Pyke, D.F., and Peterson, R.; Inventory Management and Production Planning and Scheduling; Wiley; 1998.

27. Tayur, S., Ganeshan, R., and Magazine, M.; Quantitative Models for Supply Chain Management; Kluwer's International Series; 1999.

28. Khouja, M.; The Single Period (Newsvendor) Problem: Literature Review and Suggestions for Future Research; Omega; 1999; Vol. 27: p. 537-553.

29. De Horatius, N.; Critical Determinants of Retail Execution; 2002; Harvard Business School.

30. Sahin, E., Buzacott, J., and Dallery, Y.; Impact of Inventory Record Inaccuracies on the Newsvendor with Uniformly Distributed Demands; Technical report, Laboratoire Génie Industriel, Ecole Centrale Paris; 2005.

\section{Appendix 1}

The expected total cost function associated with Model 1 will be expressed by:

$$
\begin{gathered}
\mathrm{C}_{1}(\mathrm{Q})=\mathrm{h} . \mathrm{E}\left[\operatorname{Max}\left(0, \mathrm{Q}_{\mathrm{PH}}-\operatorname{Min}\left(\mathrm{D}, \mathrm{Q}_{\mathrm{IS}}\right)\right)\right]+\mathrm{u}_{1} . \mathrm{E}\left[\operatorname{Max}\left(0, \mathrm{D}-\mathrm{Q}_{\mathrm{IS}}\right)\right] \\
+\mathrm{u}_{2} . \mathrm{E}\left[\operatorname{Max}\left(0, \operatorname{Min}\left(\mathrm{D}, \mathrm{Q}_{\mathrm{IS}}\right)-\mathrm{Q}_{\mathrm{PH}}\right)\right]
\end{gathered}
$$

By assuming that $Q_{I S}$ can take values that are either less or more than $Q_{P H}$, we obtain the following exact formulation of the expected cost function: 
24 Journal of Decision Systems. Vol. - No.

$$
\begin{aligned}
& C_{1}(Q)=\int_{p=1}^{\infty} \int_{x=p . Q}^{\infty}\left(u_{1} \cdot(x-p \cdot Q)+u_{2} \cdot(p \cdot Q-Q)\right) f(x) g(p) d x d p \quad Q_{P H} \leq Q_{I S} \leq D \\
& +\int_{p=-\infty}^{1} \int_{x=Q}^{\infty}\left(u_{1} \cdot(x-p \cdot Q)+h .(Q-p . Q)\right) f(x) g(p) d x d p \quad-Q_{I S} \leq Q_{P H} \leq D \\
& +\int_{p=-\infty}^{1} \int_{x=p . Q}^{Q}\left(u_{1} \cdot(x-p \cdot Q)+h \cdot(Q-p \cdot Q)\right) f(x) g(p) d x d p \quad-Q_{I S} \leq D \leq Q_{P H} \\
& +\int_{p=1}^{\infty} \int_{x=Q}^{p \cdot Q} u_{2} .(x-Q) f(x) g(p) d x d p \quad Q_{P H} \leq D \leq Q_{I S} \\
& +\int_{p=-\infty x=-\infty}^{1} \int_{\infty}^{p . Q} h \cdot(Q-x) f(x) g(p) d x d p \quad D \leq Q_{I S} \leq Q_{P H} \\
& +\int_{p=1}^{\infty} \int_{x=0}^{Q} h \cdot(Q-x) f(x) g(p) d x d p \quad D \leq Q_{P H} \leq Q_{I S}
\end{aligned}
$$

where $\mathrm{x}$ is the random variable that represents demand, $\mathrm{f}(\mathrm{x})$ is the probability density function of $x$ and $g(p)$ is the probability density function of $p$. 